OPEN ACCESS

Edited by: Qiongjie Zhou,

Fudan University, China

Reviewed by:

Xiaocan Lei,

University of South China, China

Shun Zhang,

Affiliated Hospital of Guilin Medical

University, China

Tao Tang,

The Chinese University of Hong Kong,

China

${ }^{*}$ Correspondence:

Hongying Hou

houhongying123@126.com

Yuzhu Yin

yzzst2011@163.com

${ }^{\dagger}$ These authors have contributed equally to this work

Specialty section: This article was submitted to Reproduction,

a section of the journal Frontiers in Endocrinology

Received: 01 April 2021 Accepted: 24 May 2021

Published: 10 June 2021

Citation:

Han Z, Zhang Y, Li X, Chiu W-H, Yin Y and Hou $H$ (2021) Investigation Into the Predictive Potential of Three-

Dimensional Ultrasonographic

Placental Volume and Vascular Indices in Gestational Diabetes Mellitus.

Front. Endocrinol. 12:689888. doi: 10.3389/fendo.2021.689888

\section{Investigation Into the Predictive Potential of Three-Dimensional Ultrasonographic Placental Volume and Vascular Indices in Gestational Diabetes Mellitus}

\author{
Zhenyan Han $^{1 \dagger}$, Yuan Zhang ${ }^{1 \dagger}$, Xuelan $\mathrm{Li}^{1,2}$, Wei-Hsiu Chiu ${ }^{3,4}$, Yuzhu Yin ${ }^{1 *}$ \\ and Hongying Hou ${ }^{1 *}$

\begin{abstract}
1 Department of Obstetrics and Gynecology, Third Affiliated Hospital of Sun Yat-sen University, Guangzhou, China, 2 Health Care Center of Minzhi Community, Shenzhen, China, ${ }^{3}$ Department of Obstetrics and Gynecology, Guangzhou Children's Peace Hospital, Xuzhou, China
\end{abstract} \\ iBorn Women's Hospital, Guangzhou, China, ${ }^{4}$ Department of Ultrasound in Obstetrics and Gynecology, Xuzhou Women and
}

Background: The use of ultrasonography in pregnancies complicated with gestational diabetes mellitus (GDM) can vary according to clinical practice. This study aims to compare the changes of placental volume (PV) and vascular indices measured by three-dimensional (3D) Power Doppler between pregnant women with and without GDM.

Materials and Methods: This was a prospective study of singleton pregnancies who took the early nuchal translucency examination from January 2018 to September 2019. Data on PV and vascular indices including vascularization index (VI), flow index (FI), and vascularization flow index (VFI) between pregnant women with and without GDM were measured by 3D Power Doppler ultrasound machine. Univariate and multivariate logistic regression determined the association between risk factors and GDM. Receiver operating characteristic (ROC) and area under the ROC curve (AUC) were applied to evaluate the diagnostic value of different parameters for GDM.

Results: Of the 141 pregnant women enrolled, 35 developed GDM and 106 did not. The maternal age and gravida in the GDM group were significantly higher than that in the nonGDM group. The PV, VI, FI, and VFI in the GDM group were significantly lower than that in the non-GDM group. There were no significant differences in other clinical parameters between the two groups. After adjustments in multivariate logistic regression analysis, significant differences were observed in $\mathrm{VI}$ [odds ratio $(\mathrm{OR})=0.98,95 \%$ confidence interval $(\mathrm{Cl})=$ 0.951-1.002], FI (OR = 0.93, $955 \mathrm{Cl}: 0.86-1.00)$, and VFI (OR $=0.67,95 \% \mathrm{Cl}=0.52-0.87)$. $\mathrm{ROC}$ analysis indicated that the combination of maternal age, gravida, PV, and VFI was more accurate as a marker for detecting GDM than the PV, VI, FI, or VFI alone.

Conclusions: The 3D ultrasonography results suggest that PV and vascular indices $\mathrm{VI}$, $\mathrm{FI}$, and VFI) during the first trimester may serve as potential markers for GDM diagnosis. 
The combination of maternal age, gravida, and sonographic markers may have good diagnostic values for GDM, which should be confirmed by further investigations.

Keywords: gestational diabetes mellitus, three-dimensional power Doppler, placental volume, vascularization index, flow index, vascularization flow index

\section{INTRODUCTION}

Gestational diabetes mellitus (GDM) is featured by abnormal glucose intolerance during pregnancy (1). GDM affects about 7\% of pregnancies, where advanced maternal age, obesity, previous history of GDM, previously giving birth to macrosomic infants, and family history of diabetes mellitus are regarded as major risk factors (2-4). Oral glucose tolerance test is often used for screening GDM during 24-28 gestational weeks $(5,6)$. However, GDM screening at 24-28 gestational weeks is too late for the physicians to make dietary or pharmacology therapy, which may ultimately affect placental integrity and fetal growth $(7,8)$. Thus, identifying novel markers for early screening of GDM is of great clinical significance.

Although clinical manifestations of GDM usually occur in the second or third trimester, evidence of abnormal placental development from as early as the first trimester has been reported, and dysregulation of various hormones and cytokines caused by GDM during early pregnancy has been reported to lead to placental dysfunction (9). Furthermore, increases in inflammatory markers due to persistent hyperglycemia can cause damage within the placenta such as villous maturation, vascularization, and branching (10). Ultrasonography is a non-invasive, readily available tool to examine and assess the fetus, which is helpful in instituting early therapeutic interventions for pregnancies complicated by diabetes (11). Improvements in three-dimensional (3D) ultrasonography have pointed out the role of placental volume (PV) and the potentially associated factors contributing to pregnancy complications. Pala et al., used Virtual Organ Computer-aided AnaLysis (VOCAL) to evaluate PV and placental mean gray value, and found that PV was significantly increased in GDM, whereas mean gray values did not alter (12). Saha et al., observed that the placentae in GDM were significantly bigger in size, weight, volume, area, thickness, diameter, and circumference than those in normal pregnant women, and there was significant increase in villous edema, fibrin deposition, calcification, and congestion of blood vessels in GDM (13). Studies from Wong et al., showed that placental vascular indices can provide an insight into placental vascularization in GDM during early pregnancy, and vascularization flow index (VFI) rather than placental volume may be a sensitive sonographic marker in the first trimester of GDM placentas (14), which still need to be confirmed with larger sample size. Desoye et al., proposed that the placentae in diabetic pregnancies increased levels of thromboxane and tumor necrosis factor alfa leading to vasoconstriction that may contribute to the decrease in vascularization index (VI) and VFI (15).

Based on the above evidence, the association between morphometric changes of placenta and GDM during early pregnancy remains elusive. Therefore, this study aims to determine the PV and vascular indices [vascularization index (VI), flow index (FI), and VFI] by using VOCAL during pregnancy, and to explore the association between these sonographic markers in combination with other clinical parameters and GDM. The present study may identify sensitive sonographic markers for the early diagnosis of GDM, which may be important to avoid the potential complications of GDM.

\section{MATERIALS AND METHODS}

This prospective study of singleton pregnancies who took the early nuchal translucency examination was performed at Guangzhou iBorn Women's Hospital from January 2018 to September 2019. A total of 161 pregnant women received ultrasonographic examination during $11^{+0}$ and $13^{+6}$ weeks. The inclusion criteria were: (1) gestational age between $11+{ }^{0}$ and $13+{ }^{6}$ weeks; (2) crown-lump length (CRL) between 45 and $80 \mathrm{~mm}$; (3) singleton pregnancy. The exclusion criteria were: (1) fetal chromosomal or structural anomalies detected during karyotyping or sonographic examinations; (2) multifetal pregnancy; (3) history of hypertension or preeclampsia, thyroid disease, chronic kidney disease, autoimmune disease, or a diagnosis of these diseases in the current pregnancy; (4) pregestational diabetes mellitus; (5) longterm use of aspirin or glucocorticoids. This study was approved by the Ethical Committee of Guangzhou iBorn Women's Hospital, and each patient signed the written informed consent.

A Voluson E8 ultrasound machine (GE Medical Systems, Milwaukee, WI, USA) with a 1-6 MHz transabdominal RAB6-1D probe was used in this study. The ultrasound scan was performed according to the ISUOG practice guidelines (16). The nuchal translucency (NT), biparietal diameter (BPD), CRL, abdominal circumference (AC), femur length (FL), uterine artery doppler pulsatility index (UTPI), uterine artery resistance index (UTRI), VI, FI, and VFI were measured between $11^{+0}$ and $13^{+6}$ gestational weeks.

To obtain optimal PV, the probe was placed along the alignment of the placenta. Each woman was asked to hold her breath for $10 \mathrm{~s}$, and the margin of the placenta was outlined to obtain its maximum area. This procedure was repeated six times after rotating the probe 30 degrees around the axis each time to acquire the full volume of the placenta. For laterally and posteriorly located placentas, the position of the probe was adjusted to fit the placental alignment as far as possible to obtain the optimal volume (Figure 1). All of the examined cases were measured using the same ultrasound instrument settings (Power Doppler map, 6; frequency, low; smoothing, rise 2/fall 4; flow resolution, mild 1; line density, 7; balance, 210; ensemble, 10; line filter, 3; artifact suppression, on; quality, high1; wall motion filter, med 1; pulse repetition frequency, $1.3 \mathrm{kHz}$ ). All the ultrasound scans were performed by the same examiner, and the placental vascular indices and PV were measured twice to inspect the intrarater reliability. 

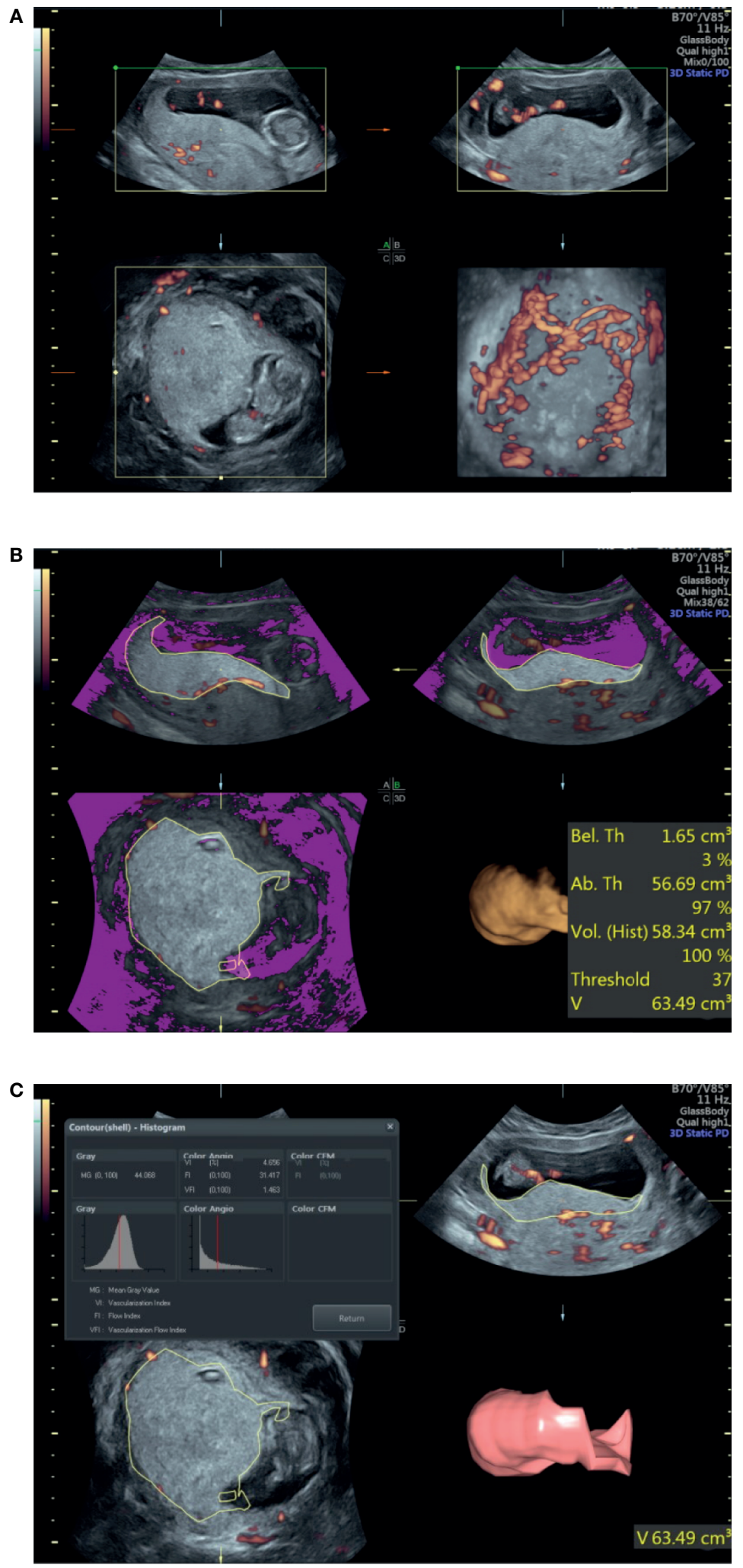

FIGURE 1 | Ultrasound examination of the placental volume and vascular perfusion. (A) Placental volume as determined by three-dimensional power Doppler. (B) Measurement of placental volume by VOCAL. (C) Placental vascular indices determined by three-dimensional power Doppler. 
The three vascular indices (VI, FI, and VFI) were developed through specific algorithms based on signal intensity and the relative proportion of color voxels (three-dimensional pixels) within the defined volume, where VFI is a combination of VI and FI information (17). The placental vascular indices were automatically calculated using VOCAL ${ }^{\mathrm{TM}}$ imaging software (GE Medical Systems) and expressed on a scale of 0-100 (Figure 1).

All pregnant women were followed up after delivery. Screening for GDM was universally performed at 24-28 gestational weeks according to Guideline for Diagnosis and Treatment of Gestational Diabetes Mellitus (2014) (6). The GDM is diagnosed if any of the oral glucose tolerance test plasma glucose values meets or exceeds the following cut-off values: $5.1 \mathrm{mmol} / \mathrm{L}$ at fasting; $10.0 \mathrm{mmol} / \mathrm{L}$ at $1 \mathrm{~h}$; and $8.5 \mathrm{mmol} / \mathrm{L}$ at $2 \mathrm{~h}$. The follow-up data were collected as follows: pregnant complications, gestational age, modes of delivery, 1 and 5 min Apgar scores, birth weight, placental weight, and placental volume after delivery. The placental weight and placental volume after delivery were determined according to previous methods (18).

All the data analyses were performed using SPSS software (version 22.0, IBM, Armonk, USA). The normality of the data was examined by the Kolmogorov-Smirnov test. The Chi-square test was used for categorical variables. The Student's t-test was used to evaluate differences in the continuous data between nonGDM and GDM group, and the continuous data were presented as mean \pm standard deviation. Univariate and multivariate logistic regression analyses of individual confounding factor were also performed. Receiver operating characteristic (ROC) and the area under the ROC curve (AUC) were applied to evaluate the diagnostic value and the Youden index is a measure of a diagnostic test's ability to balance sensitivity (detecting disease) and specificity (detecting health or no disease) (19). Youden index was applied to determine the optimal cut-off points of different parameters in the diagnosis of GDM. $\mathrm{P}<0.05$ was considered statistical significance.

\section{RESULTS}

\section{Clinical Characteristics of Pregnant Women in the Non-GDM and GDM Group}

In this study, a total of 161 pregnant women received ultrasonographic examination. Among these pregnancies, four women had miscarriage during the second trimester; nine women had pregnant complications (hypertension, preeclampsia, or thyroid disease); seven women lost to follow-up. Thus, 106 pregnant women without GDM and 35 pregnant women with GDM were included in this study. As shown in Table 1, the maternal age and gravida in the GDM group were significantly higher than that in the non-GDM group (Table 1). For the ultrasonographic examination, no significant difference was detected in NT, BPD, CRL, AC, FL, UTPI, and UTRI between non-GDM and GDM group (Table 1). There was no significant difference in gestational age at delivery, Caesarean section, and premature birth rates between non-GDM and GDM group (Table 1). For the newborns, no significant difference was identified in birth weight, placental weight, PV

TABLE 1 | Comparison of clinical characteristics between non-GDM group and GDM group.

\begin{tabular}{|c|c|c|c|}
\hline & Non-GDM group $(n=106)$ & GDM group $(n=35)$ & $P$ value \\
\hline \multicolumn{4}{|l|}{ Maternal } \\
\hline Maternal age (years old) & $30.04 \pm 4.24$ & $32.40 \pm 4.56$ & 0.004 \\
\hline $\mathrm{BMI}\left(\mathrm{kg} / \mathrm{m}^{2}\right)$ & $21.36 \pm 2.54$ & $22.34 \pm 2.98$ & 0.062 \\
\hline Gravida & $1.88 \pm 1.03$ & $2.34 \pm 1.37$ & 0.035 \\
\hline \multicolumn{4}{|l|}{ Parity } \\
\hline Primiparity & 61 & 17 & 0.4336 \\
\hline Multiparity & 45 & 18 & \\
\hline \multicolumn{4}{|l|}{ Ultrasonography } \\
\hline $\mathrm{NT}(\mathrm{mm})$ & $1.45 \pm 0.40$ & $1.35 \pm 0.38$ & 0.972 \\
\hline $\mathrm{BPD}(\mathrm{cm})$ & $2.04 \pm 0.26$ & $2.03 \pm 0.27$ & 0.706 \\
\hline $\mathrm{CRL}(\mathrm{cm})$ & $6.29 \pm 0.82$ & $6.06 \pm 0.79$ & 0.160 \\
\hline $\mathrm{AC}(\mathrm{cm})$ & $6.19 \pm 0.82$ & $5.95 \pm 0.86$ & 0.128 \\
\hline $\mathrm{FL}(\mathrm{cm})$ & $0.75 \pm 0.20$ & $0.72 \pm 0.22$ & 0.335 \\
\hline UTPI & $1.43 \pm 0.51$ & $1.40 \pm 0.45$ & 0.776 \\
\hline UTRI & $0.67 \pm 0.13$ & $0.64 \pm 0.16$ & 0.305 \\
\hline \multicolumn{4}{|l|}{ Outcomes } \\
\hline Gestational age at delivery (weeks) & $39.31 \pm 1.32$ & $38.89 \pm 1.83$ & 0.141 \\
\hline Caesarean section (\%) & $36.79 \%(39 / 106)$ & $40.00 \%(14 / 35)$ & 0.841 \\
\hline Premature birth (\%) & $5.66 \%(6 / 106)$ & $2.86 \%(1 / 35)$ & 0.681 \\
\hline Birth weight (g) & $3203 \pm 394$ & $3229 \pm 496$ & 0.182 \\
\hline Placental weight (g) & $561.50 \pm 83.74$ & $548.56 \pm 68.31$ & 0.471 \\
\hline Placental volume after delivery $\left(\mathrm{cm}^{3}\right)$ & $462.83 \pm 123.65$ & $462.55 \pm 104.36$ & 0.991 \\
\hline 1 min Apgar score & $9.95 \pm 0.25$ & $10.00 \pm 0.00$ & 0.275 \\
\hline 5 min Apgar score & $10.00 \pm 0.00$ & $10.00 \pm 0.00$ & - \\
\hline
\end{tabular}

AC, abdominal circumference; BMI, body mass index; BPD, biparietal diameter; CRL, crown-rump length; FL, Femur length; GDM, gestational diabetes mellitus; NT, nuchal translucency; UTPI, uterine artery doppler pulsatility index; UTRI, uterine artery resistance index. 
after delivery, 1 and 5 min Apgar scores, between non-GDM and GDM group (Table 1).

\section{Comparison of Placental Volume and Vascular Perfusion Between Non-GDM Group and GDM Group}

The gestational age at ultrasound examination was $12.76 \pm 0.48$ and $12.64 \pm 0.43$ weeks in non-GDM and GDM group, respectively (Table 2 ). In the non-GDM group, $\mathrm{PV}\left(\mathrm{cm}^{3}\right), \mathrm{VI}$, FI, and VFI were $50.49 \pm 18.53,13.08 \pm 6.30,31.71 \pm 5.71$, and 4.15 \pm 2.17 , respectively (Table 2 ); in the GDM group, the PV $\left(\mathrm{cm}^{3}\right)$, VI, FI, and VFI were 43.20 $\pm 14.07,9.49 \pm 6.46,29.18 \pm 5.46$, and $2.82 \pm 2.05$ (Table 2). PV, VI, FI, and VFI in the GDM group were significantly lower than that in the non-GDM group (Table 2). The distribution of pregnant women based on maternal age, maternal BMI, gestational age at examination, PV, VI, FI, VFI, gestational age at delivery, placental weight, and PV after delivery

TABLE 2 | Comparison of placental volume and vascular perfusion between non-GDM group and GDM group.

\begin{tabular}{lccc}
\hline & $\begin{array}{c}\text { Non-GDM } \\
\text { group } \\
(\mathbf{n}=\mathbf{1 0 6})\end{array}$ & $\begin{array}{c}\text { GDM group } \\
(\mathbf{n}=\mathbf{3 5})\end{array}$ & $\boldsymbol{P}$ value \\
\hline Gestational age at examination & $12.76 \pm 0.48$ & $12.64 \pm 0.43$ & 0.184 \\
(weeks) & $50.49 \pm 18.53$ & $43.20 \pm 14.07$ & 0.025 \\
PV $\left(\mathrm{cm}^{3}\right)$ & $13.08 \pm 6.30$ & $9.49 \pm 6.46$ & 0.001 \\
VI $(\%)$ & $31.71 \pm 5.71$ & $29.18 \pm 5.46$ & 0.023 \\
FI & $4.15 \pm 2.17$ & $2.82 \pm 2.05$ & $<0.001$ \\
VFI & & &
\end{tabular}

FI, flow index; GDM, gestational diabetes mellitus; PV, placental volume; VFI, vascularization-flow index; VI, vascularization index. was shown in Figure 2. In terms of maternal age, the distribution of maternal age was mainly between 25 and 35 years old; while the proportion of patients $>35$ years old was higher in the GDM group (Figure 2). The distribution of BMI, PV, and FI was mainly between 18 and $24 \mathrm{~kg} / \mathrm{m}^{2}, 30-60 \mathrm{~cm}^{3}$, and 20-40, respectively (Figure 2). In terms of VFI, the distribution of VFI $<2$ in GDM group was higher than that in the non-GDM group (Figure 2).

\section{Univariate and Multivariate Logistic Regression Analyses of Clinical Parameters Associated With GDM}

Univariate and multivariate analyses were performed to determine the association between clinical parameters and GDM. The univariate analysis showed that maternal age [odds ratio $(\mathrm{OR})=1.13,95 \%$ confidence interval $(\mathrm{CI})=1.03-1.23$, $\mathrm{P}=0.008]$, gravida $(\mathrm{OR}=2.55,95 \% \mathrm{CI}=1.12-5.80, \mathrm{P}=0.0026)$, $\mathrm{PV}(\mathrm{OR}=0.97,95 \% \mathrm{CI}=0.95-1.00, \mathrm{P}=0.038), \mathrm{VI}(\mathrm{OR}=0.90,95 \%$ $\mathrm{CI}=0.83-0.97, \mathrm{P}=0.006), \mathrm{FI}(\mathrm{OR}=0.92,95 \% \mathrm{CI}=0.86-0.99$, $\mathrm{P}=0.026)$, and VFI $(\mathrm{OR}=0.68,95 \% \mathrm{CI}=0.53-0.87, \mathrm{P}=0.002)$ were significantly associated with GDM (Table 3). Multivariate analysis revealed that VI $(\mathrm{OR}=0.89,95 \% \mathrm{CI}=0.83-0.96$, $\mathrm{P}=0.003)$, FI (OR: $0.93,95 \% \mathrm{CI}=0.86-1.00, \mathrm{P}=0.038)$, and VFI $(\mathrm{OR}=0.67,95 \% \mathrm{CI}=0.52-0.87, \mathrm{P}=0.002)$ were significantly associated with GDM (Table 3); while PV (OR $=0.98,95 \%$ $\mathrm{CI}=0.95-1.00, \mathrm{P}=0.076$ ) had no effect on GDM (Table 3).

\section{Diagnostic Values of PV, VI, FI, and VFI for GDM}

Subsequently, ROC curves were used to evaluate the ability of $\mathrm{PV}$, VI, FI, and VFI for diagnosing GDM. The ROC analysis revealed that PV, VI, FI, and VFI yielded an AUC of 0.63, 0.69,
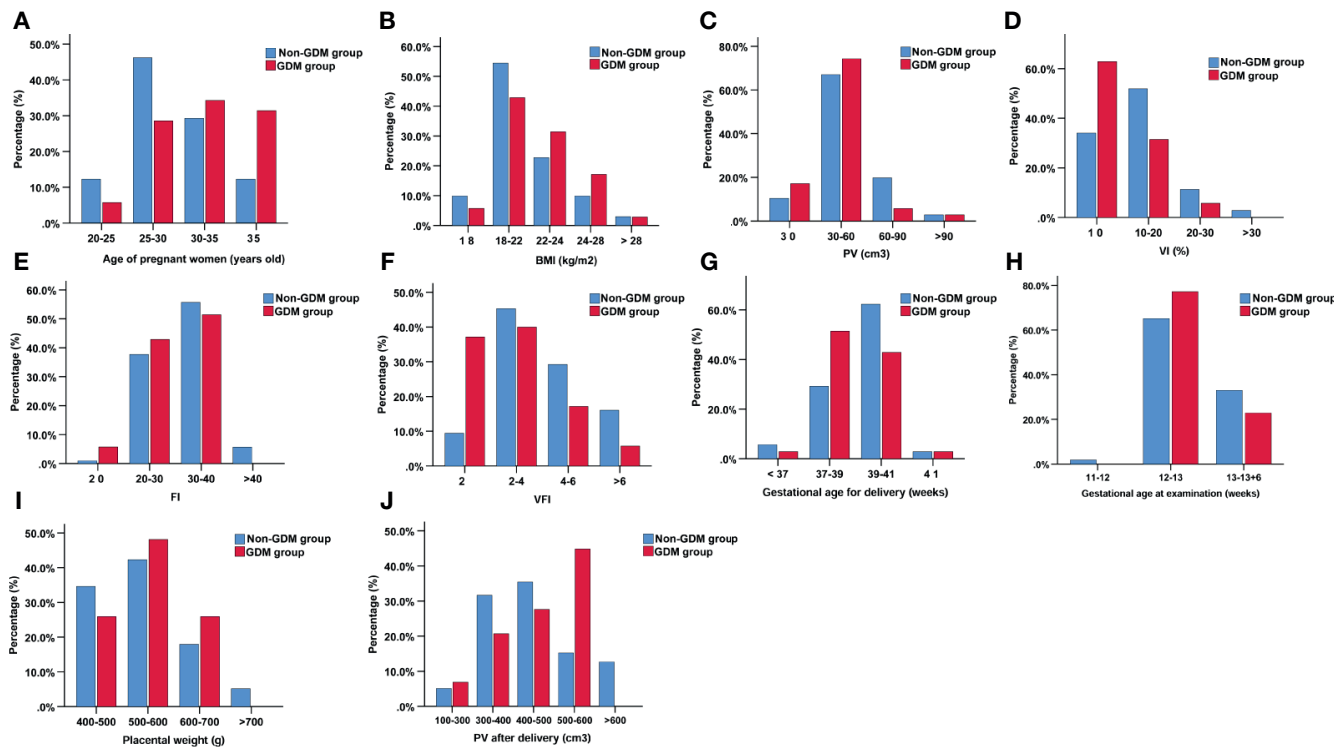

FIGURE 2 | The distribution of pregnant women based on age of pregnant women (A), BMI (B), PV (C), VI (\%) (D), FI (E), VFI (F), gestational age for delivery (G), gestational age at examination (H), placental weight (I), and PV after delivery (J). 
TABLE 3 | Univariate and multivariate logistic regression analysis of clinical parameters associated with GDM.

\begin{tabular}{|c|c|c|c|c|c|c|}
\hline \multirow[t]{2}{*}{ Variables } & \multicolumn{3}{|c|}{ Univariate analysis } & \multicolumn{3}{|c|}{ Multivariate analysis } \\
\hline & OR & $95 \% \mathrm{Cl}$ & $P$ value & OR & $95 \% \mathrm{Cl}$ & $P$ value \\
\hline Age & 1.13 & $1.03-1.23$ & 0.008 & & & \\
\hline Gravida & 2.55 & $1.12-5.80$ & 0.026 & & & \\
\hline $\mathrm{PV}\left(\mathrm{cm}^{3}\right)$ & 0.97 & $0.95-1.00$ & 0.038 & 0.98 & $0.95-1.00$ & 0.076 \\
\hline VI (\%) & 0.90 & $0.83-0.97$ & 0.006 & 0.89 & $0.83-0.96$ & 0.003 \\
\hline $\mathrm{FI}$ & 0.92 & $0.86-0.99$ & 0.026 & 0.93 & $0.86-1.00$ & 0.038 \\
\hline VFI & 0.68 & $0.53-0.87$ & 0.002 & 0.67 & $0.52-0.87$ & 0.002 \\
\hline
\end{tabular}

Cl, confidence interval; Fl, flow index; OR, odds ratio; PV, placental volume; VFI, vascularization-flow index; VI, vascularization index.

0.61 , and 0.71 , respectively (Table 4 and Figure 3 ). In addition, based on the Youden index, the results indicated that VFI (Youden index $=0.40$ ) had the best diagnostic value for GDM when compared to VI (Youden index $=0.38$ ), PV (Youden index $=0.23$ ), and FI (Youden index $=0.20$; Table 4 and Figure 3). As maternal age and gravida were potential risk factors for GDM in our cohort, we combined maternal age, gravida, PV, and VFI by using the regression model. The ROC analysis revealed an AUC of 0.76 (95\% CI $=0.66-0.86, \mathrm{P}<0.001$; Table 4 and Figure 3). ROC analysis indicated that the combination of maternal age, gravida, PV and VFI was more accurate as a marker for detecting GDM than the PV, VI, FI, or VFI alone (Table 4 and Figure 3E).

\section{DISCUSSION}

According to the demographic data, advanced maternal age is one of the risk factors for GDM. Schaefer et al. found that women older than 35 years old had 3.95-fold increased risk of GDM compared with women aged between 16 and 25 years old (20). A study by analyzing the prevalence of GDM in the USA between 2007 and 2014 indicated that older age was one of the risk factors associated with GDM (21). Shan et al., found that there was a 23 -fold risk for mothers with advanced maternal age to be diagnosed with GDM in a retrospective cohort study from China (22). Khalil et al., analyzed a population of 22,933 pregnancies, and demonstrated that advanced maternal age was a risk factor for GDM (23). Our results consistently showed that the maternal age in the GDM group was higher than that in the non-GDM group, and maternal age was included in the subsequent ROC analysis.
In this study, we used the VOCAL to determine the PV and placental vascular indices (VI, FI, and VFI). Our results showed that the PV in the GDM group was significantly smaller than that in the non-GDM group during early pregnancy. However, Wong et al., showed that the PV was similar between GDM and non-GDM group during the first trimester, while it was significantly increased in the GDM group during the second trimester (14). Moreover, studies demonstrated that placental calcification and volume increased with advancing gestation in pre-gestational diabetic placentae $(12,24)$. On the other hand, no significant difference was detected in the PV and placental weight after delivery. These discrepancies may be to the small sample size and the variations in determine the PV after delivery, and future studies should include large samples to confirm our findings. In the analysis of VI, FI, and VFI, previous studies from Hafner et al., showed that VI, FI, and VFI could be used for a quick and reliable first trimester assessment of severe pregnancy risks (25). Consistently, Wong et al., showed that VI, FI, and VFI were significantly lower in the GDM group during the first and second trimesters (14). In addition, VI, FI, and VFI were significantly lower in diabetic pregnancies between 35 and 40 gestational weeks (24). Consistently, our results showed that the above three vascular indices were significantly lower in the GDM group during the early pregnancy. The above results indicated that there was a remarkable reduction in VI, FI, and VFI during GDM.

Based on the logistic regression analysis, our results indicated that reduced PV, VI, FI, and VFI were associated with the increased risk of GDM, which was consistent with the findings from Wong et al. (14). In addition, PV and vascular indices during early pregnancy could be used to predict the pregnant complications (25). Although the PV increases with the advancement in gestational age, the placental vascular index of pregnant women with GDM still decreases during the third

TABLE 4 | Diagnostic values of PV, VI, FI, and VFI for GDM.

\begin{tabular}{|c|c|c|c|c|c|c|c|c|c|}
\hline Variables & AUC & Sensitivity (\%) & Specificity (\%) & Cut-off value & $95 \% \mathrm{Cl}$ & LR+ & LR- & Youden index & $P$ value \\
\hline $\mathrm{PV}\left(\mathrm{cm}^{3}\right)$ & 0.63 & 65.71 & 57.55 & 44.90 & $0.52-0.73$ & 1.55 & 0.60 & 0.23 & 0.017 \\
\hline VI (\%) & 0.69 & 60.00 & 78.30 & 8.22 & $0.57-0.80$ & 2.77 & 0.51 & 0.38 & 0.002 \\
\hline $\mathrm{Fl}$ & 0.61 & 91.43 & 28.30 & 34.93 & $0.50-0.71$ & 1.28 & 0.30 & 0.20 & 0.053 \\
\hline VFI & 0.71 & 60.00 & 80.19 & 2.62 & $0.60-0.82$ & 3.03 & 0.50 & 0.40 & $<0.001$ \\
\hline Regression model & 0.761 & 62.26 & 82.86 & - & $0.66-0.86$ & 3.24 & 0.46 & 0.45 & $<0.001$ \\
\hline
\end{tabular}

AUC, area under the curve; FI, flow index; LR+, positive likelihood ratio; LR-, negative likelihood ratio; OR, odds ratio; PV, placental volume; VFI, vascularization-flow index; VI, vascularization index. 
A

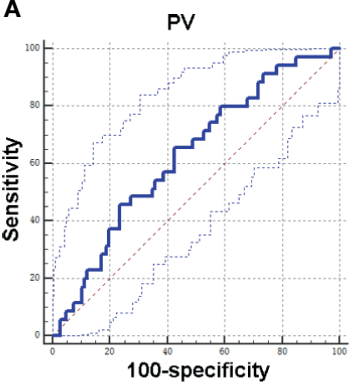

D

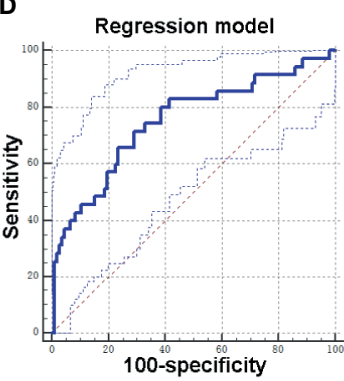

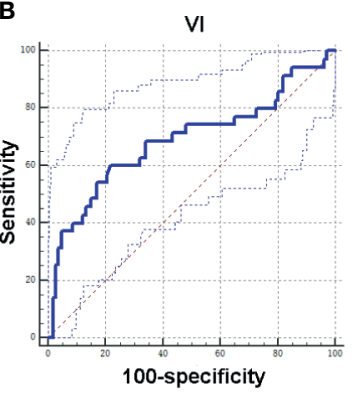

E

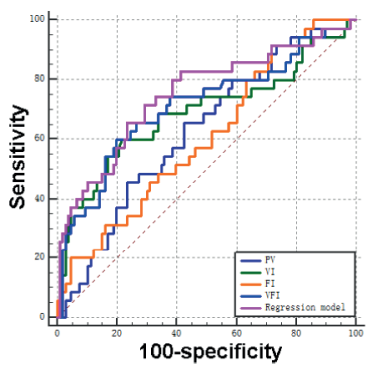

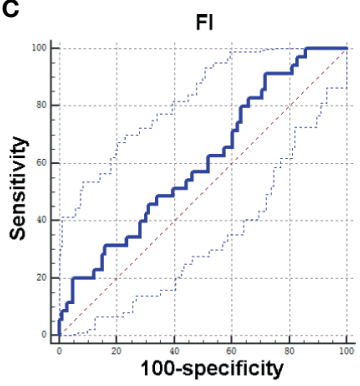

FIGURE 3 | Diagnostic values of PV, VI, FI, and VFI for GDM. (A) ROC curve of PV for distinguishing GDM patients from non-GDM patients. (B) ROC curve of VI for distinguishing GDM patients from non-GDM patients. (C) ROC curve of Fl for distinguishing GDM patients from non-GDM patients. (D) ROC curve of integrated factors using regression model for distinguishing GDM patients from non-GDM patients. (E) ROC curves of individual factor and integrated factors for distinguishing GDM patients from non-GDM patients.

trimester (14). This finding may be related to the abnormal secretion of key mediators in GDM pregnant women in the first trimester, which affects placental vascular remodeling, and finally affects the placental development, leading to increased PV and reduced vascular indices $(26,27)$. Moran et al., found that PV was significantly larger at all stages of gestation from 12 weeks (24). In our study, the PV was mainly determined between $11^{+0}$ and $13^{+6}$ weeks, when the changes in placenta have taken place, thus, suggesting the reliability of our findings.

This study also determined diagnostic potentials of PV, VI, FI, VFI in GDM. According to the results of the ROC curve of each parameter, the AUC of VFI curve is the largest, indicating good predictive value, and its sensitivity and specificity are 60.00 and $80.19 \%$, respectively, which means that the diagnosis rate is high and the missed diagnosis rate is low. In addition, Youden index is the highest for VFI, indicating that it has good clinical practical value. The AUC values of PV, VI, and FI were less than 0.7 , indicating that its predictive value is low. FI has the highest sensitivity, but low specificity, which indicates that its misdiagnosis rate is high. Our results were consistent with previous studies showing that VFI may be a more sensitive sonographic marker than VI and FI in the first trimester of GDM placentas (14). Our results of combined maternal age, gravida, PV, and VFI by using the regression model revealed that the combination of these parameters was more accurate as a marker for detecting GDM than the PV, VI, FI, or VFI alone, suggesting that the maternal age, gravida, PV, and VFI may representative important diagnostic markers for GDM.
This study is subjected to several limitations. First of all, the present study is limited to small sample size, and larger population of pregnant women may be included for analysis in the future. Secondly, this study was a single-center prospective study, which may lead to study bias. Multiple-center studies should be considered, in order to confirm our findings. Thirdly, how PV and vascular indices correlate with GDM remains unknown, which still requires the mechanistic investigations.

In conclusion, the $3 \mathrm{D}$ ultrasonography results suggest that $\mathrm{PV}$ and vascular indices (VI, FI, and VFI) during the first trimester may serve as potential markers for GDM diagnosis. The combination of maternal age, gravida, and sonographic markers may have good diagnostic values for GDM, which should be confirmed by further investigations.

\section{DATA AVAILABILITY STATEMENT}

The original contributions presented in the study are included in the article/supplementary material. Further inquiries can be directed to the corresponding authors.

\section{ETHICS STATEMENT}

This study was approved by the Ethical Committee of Guangzhou iBorn Women's Hospital, and each patient signed 
the written informed consent. The patients/participants provided their written informed consent to participate in this study.

\section{AUTHOR CONTRIBUTIONS}

$\mathrm{HH}, \mathrm{YY}$, and $\mathrm{W}-\mathrm{HC}$ conceptualized the study and were involved in study design. $\mathrm{ZH}$ and $\mathrm{YZ}$ collected the data and performed the follow-up. XL and W-HC performed the data analysis. $\mathrm{ZH}$ and $\mathrm{YZ}$

\section{REFERENCES}

1. Saravanan P. Gestational Diabetes: Opportunities for Improving Maternal and Child Health. Lancet Diabetes Endocrinol (2020) 8(9):793-800. doi: 10.1016/S2213-8587(20)30161-3

2. Chiefari E, Arcidiacono B, Foti D, Brunetti A. Gestational Diabetes Mellitus: An Updated Overview. J Endocrinol Invest (2017) 40(9):899-909. doi: 10.1007/s40618-016-0607-5

3. Coustan DR. Gestational Diabetes Mellitus. Clin Chem (2013) 59(9):1310-21. doi: 10.1373/clinchem.2013.203331

4. Johns EC, Denison FC, Norman JE, Reynolds RM. Gestational Diabetes Mellitus: Mechanisms, Treatment, and Complications. Trends Endocrinol Metab (2018) 29(11):743-54. doi: 10.1016/j.tem.2018.09.004

5. Spaight C, Gross J, Horsch A, Puder JJ. Gestational Diabetes Mellitus. Endocr Dev (2016) 31:163-78. doi: 10.1159/000439413

6. Juan J, Yang H. Prevalence, Prevention, and Lifestyle Intervention of Gestational Diabetes Mellitus in China. Int J Environ Res Public Health (2020) 17(24):9517. doi: 10.3390/ijerph17249517

7. Kc K, Shakya S, Zhang H. Gestational Diabetes Mellitus and Macrosomia: A Literature Review. Ann Nutr Metab (2015) 66(Suppl 2):14-20. doi: 10.1159/000371628

8. McIntyre HD, Catalano P, Zhang C, Desoye G, Mathiesen ER, Damm P. Gestational Diabetes Mellitus. Nat Rev Dis Primers (2019) 5(1):47. doi: 10.1038/s41572-019-0098-8

9. Plows JF, Stanley JL, Baker PN, Reynolds CM, Vickers MH. The Pathophysiology of Gestational Diabetes Mellitus. Int J Mol Sci (2018) 19 (11):3342. doi: 10.3390/ijms19113342

10. Huynh J, Dawson D, Roberts D, Bentley-Lewis R. A Systematic Review of Placental Pathology in Maternal Diabetes Mellitus. Placenta (2015) 36(2):10114. doi: 10.1016/j.placenta.2014.11.021

11. Ahmed B, Abushama M, Khraisheh M, Dudenhausen J. Role of Ultrasound in the Management of Diabetes in Pregnancy. J Matern Fetal Neonatal Med (2015) 28(15):1856-63. doi: 10.3109/14767058.2014.971745

12. Pala HG, Artunc-Ulkumen B, Koyuncu FM, Bulbul-Baytur Y. ThreeDimensional Ultrasonographic Placental Volume in Gestational Diabetes Mellitus. J Matern Fetal Neonatal Med (2016) 29(4):610-4. doi: 10.3109/ 14767058.2015.1012066

13. Saha S, Biswas S, Mitra D, Adhikari A, Saha C. Histologic and Morphometric Study of Human Placenta in Gestational Diabetes Mellitus. Ital J Anat Embryol (2014) 119(1):1-9. doi: 10.13128/IJAE-14634

14. Wong $\mathrm{CH}$, Chen CP, Sun FJ, Chen CY. Comparison of Placental ThreeDimensional Power Doppler Indices and Volume in the First and the Second Trimesters of Pregnancy Complicated by Gestational Diabetes Mellitus. J Matern Fetal Neonatal Med (2019) 32(22):3784-91. doi: 10.1080/14767058.2018.1472226

15. Desoye G, Hauguel-de Mouzon S. The Human Placenta in Gestational Diabetes Mellitus. Insulin Cytokine Netw Diabetes Care (2007) 30(Suppl 2): S120-6. doi: $10.2337 / \mathrm{dc} 07-\mathrm{s} 203$

16. Salomon LJ, Alfirevic Z, Bilardo CM, Chalouhi GE, Ghi T, Kagan KO, et al. ISUOG Practice Guidelines: Performance of First-Trimester Fetal Ultrasound Scan. Ultrasound Obstet Gynecol (2013) 41(1):102-13. doi: 10.1002/uog.12342 drafted the manuscript and $\mathrm{HH}$ revised it critically. All authors contributed to the article and approved the submitted version.

\section{ACKNOWLEDGMENTS}

We would like to thank all the doctors and nurses who facilitated this study at Guangzhou iBorn Women's Hospital and the Third Affiliated Hospital of Sun Yat-Sen University.

17. Pairleitner H, Steiner H, Hasenoehrl G, Staudach A. Three-Dimensional Power Doppler Sonography: Imaging and Quantifying Blood Flow and Vascularization. Ultrasound Obstet Gynecol (1999) 14(2):139-43. doi: 10.1046/j.1469-0705.1999.14020139.x

18. Balihallimath RL, Shirol VS, Gan AM, Tyagi NK, Bandankar MR. Placental Morphometry Determines the Birth Weight. J Clin Diagn Res (2013) 7 (11):2428-31. doi: 10.7860/JCDR/2013/7478.3564

19. Martínez-Camblor P, Pardo-Fernández JC. The Youden Index in the Generalized Receiver Operating Characteristic Curve Context. Int J Biostat (2019) 15(1):3342. doi: 10.1515/ijb-2018-0060

20. Schaefer KK, Xiao W, Chen Q, He J, Lu J, Chan F, et al. Prediction of Gestational Diabetes Mellitus in the Born in Guangzhou Cohort Study, China. Int J Gynaecol Obstet (2018) 143(2):164-71. doi: 10.1002/ijgo.12627

21. Casagrande SS, Linder B, Cowie CC. Prevalence of Gestational Diabetes and Subsequent Type 2 Diabetes Among U. S Women Diabetes Res Clin Pract (2018) . 141:200-8. doi: 10.1016/j.diabres.2018.05.010

22. Shan D, Qiu PY, Wu YX, Chen Q, Li AL, Ramadoss S, et al. Pregnancy Outcomes in Women of Advanced Maternal Age: A Retrospective Cohor Study from China. Sci Rep (2018) 8(1):12239. doi: 10.1038/s41598-01829889-3

23. Londero AP, Rossetti E, Pittini C, Cagnacci A, Driul L. Maternal Age and the Risk of Adverse Pregnancy Outcomes: A Retrospective Cohort Study. BMC Pregnancy Childbirth (2019) 19(1):261. doi: 10.1186/s12884-019-2400-x

24. Moran M, Mulcahy C, Daly L, Zombori G, Downey P, McAuliffe FM. Novel Placental Ultrasound Assessment: Potential Role in Pre-Gestational Diabetic Pregnancy. Placenta (2014) 35(8):639-44. doi: 10.1016/j.placenta.2014.03.007

25. Hafner E, Metzenbauer M, Stümpflen I, Waldhör T. Measurement of Placental Bed Vascularization in the First Trimester, Using 3D-PowerDoppler, for the Detection of Pregnancies At-Risk for Fetal and Maternal Complications. Placenta (2013) 34(10):892-8. doi: 10.1016/j.placenta. 2013.06.303

26. Thadhani R, Wolf M, Hsu-Blatman K, Sandler L, Nathan D, Ecker JL. FirstTrimester Sex Hormone Binding Globulin and Subsequent Gestational Diabetes Mellitus. Am J Obstet Gynecol (2003) 189(1):171-6. doi: 10.1067/ mob.2003.343

27. Nanda S, Savvidou M, Syngelaki A, Akolekar R, Nicolaides KH. Prediction of Gestational Diabetes Mellitus by Maternal Factors and Biomarkers at 11 to 13 Weeks. Prenat Diagn (2011) 31(2):135-41. doi: 10.1002/pd.2636

Conflict of Interest: The authors declare that the research was conducted in the absence of any commercial or financial relationships that could be construed as a potential conflict of interest.

Copyright (c) 2021 Han, Zhang, Li, Chiu, Yin and Hou. This is an open-access article distributed under the terms of the Creative Commons Attribution License (CC BY). The use, distribution or reproduction in other forums is permitted, provided the original author(s) and the copyright owner(s) are credited and that the original publication in this journal is cited, in accordance with accepted academic practice. No use, distribution or reproduction is permitted which does not comply with these terms. 